\title{
EVOLUTION OF WHIRL IN FLEXIBLE ISOTROPIC ROTORS MODELLING AND EXPERIMENTAL STUDY
}

\author{
Chandan Chattoraj ${ }^{1}$
}

Abstract- Rotors are principal elements of a wide variety of machineries. Early investigators had noticed the effects of imbalance and increasing speeds on the vibrations as the rotors operated near resonance. The modern high speed trend to enhance the power-to-weight ratios has rendered the study of rotor dynamics as one of upmost importance as vibration failures may prove disastrous. Etiology of rotor's dynamic behaviour may often be counter-intuitive, especially when flexible systems with rotational asymmetry are required to operate above the primary critical speed. The intent of the present work is to investigate the evolution of the vibratory motion in a horizontal rotor with orthotropic flexible end constraint (bearing), both in the sub critical and super critical states. The analysis includes the effects of rotatory inertia, gyroscopic couple, Coriolis force and internal/external damping producing a four degree of freedom system and the method was experimentally validated on a test facility.

Keywords: asymmetric rotor bearing systems; gyroscopic couple; rotatory inertia; coriolis force; supercritical response; instability.

\section{Nomenclature:}

C : Damping coefficient (translational) (N.s/m)

$C_{0} \quad$ : Damping coefficient (rotational) (N.m-s/rad)

$\mathrm{C}_{\mathrm{x}}, \mathrm{C}_{\mathrm{y}}$ : Damping coefficients at the end bearing along $\mathrm{x}$-axis and $\mathrm{y}$ - axis (N.s/m)

$\mathrm{d} \quad$ : Diameter of the shaft $(\mathrm{m})$

$\mathrm{d}_{1} \quad$ : Diameter of the disk $(\mathrm{m})$

e $\quad$ : Eccentricity of disk mass centre (m).

$\mathrm{F}_{\mathrm{x}}, \mathrm{F}_{\mathrm{y}} \quad$ : Restoring force components along $\mathrm{x}, \mathrm{y}$-axes $(\mathrm{N})$

$\mathrm{F}_{\mathrm{xr}}, \mathrm{F}_{\mathrm{yr}}$ : Restoring force components along $\mathrm{x}_{\mathrm{r}}, \mathrm{y}_{\mathrm{r}}$ axes $(\mathrm{N})$

$\mathrm{g} \quad$ : Gravitational acceleration $\left(\mathrm{m} / \mathrm{s}^{2}\right)$

J : Moment of inertia of the disk $\left(\mathrm{m}^{4}\right)$

$K \quad$ : Lateral bending stiffness of the shaft at disk location (N/m)

$K_{0} \quad$ : Rotational bending stiffness of the shaft at disk location

$\mathrm{K}_{\mathrm{x}}, \mathrm{K}_{\mathrm{y}} \quad$ : End bearing stiffness along $\mathrm{x}, \mathrm{y}$ - axes $(\mathrm{N} / \mathrm{m})$

$\mathrm{L} \quad$ : Span of the horizontal cantilever shaft (m)

$\mathrm{m} \quad$ : Equivalent mass of the rotor system $(\mathrm{kg})$

$\mathrm{p} \quad$ : Natural frequency in bending $(\mathrm{rad} / \mathrm{s})$

$\mathrm{r}_{\mathrm{c}}, \mathrm{r}_{\mathrm{k}} \quad$ : Bearing property ratio (damping, stiffness)

$\mathrm{x}, \mathrm{y} \quad$ : Non-rotating frame of reference $(\mathrm{m})$

$\mathrm{x}_{\mathrm{r}}, \mathrm{y}_{\mathrm{r}} \quad$ : Rotating frame of reference $(\mathrm{m})$

$\dot{x}, \dot{y} \quad$ : Velocity components along $\mathrm{x}$ - and $\mathrm{y}$ - axes directions respectively $(\mathrm{m} / \mathrm{s})$

$\dot{x}_{r}, \dot{y}_{r} \quad$ : Velocity components along $\mathrm{x}_{\mathrm{r}^{-}}$and $\mathrm{y}_{\mathrm{r}^{-}}$axes directions respectively $(\mathrm{m} / \mathrm{s})$

$\theta \quad:$ Angular displacement at any instant of time ' $\mathrm{t}$ ' ( $\mathrm{rad})$

$\gamma=\frac{\omega}{p} \quad:$ Frequency ratio

$\delta \quad:$ Static deflection $(\mathrm{m})=\mathrm{mg} / \mathrm{K} .=\mathrm{g} / \mathrm{p}^{2}$

$v \quad:$ Natural frequency in wobbling $(\mathrm{rad} / \mathrm{s})$

$\zeta, \zeta_{m}, \zeta_{0}:$ Damping ratios

$\mu_{c}, \mu_{k}:$ Asymmetry at bearing (damping, stiffness)

\footnotetext{
${ }^{1}$ Department of Mechanical Engineering, Dr. B.C. Roy Engineering College, Durgapur, West Bengal, India.
} 


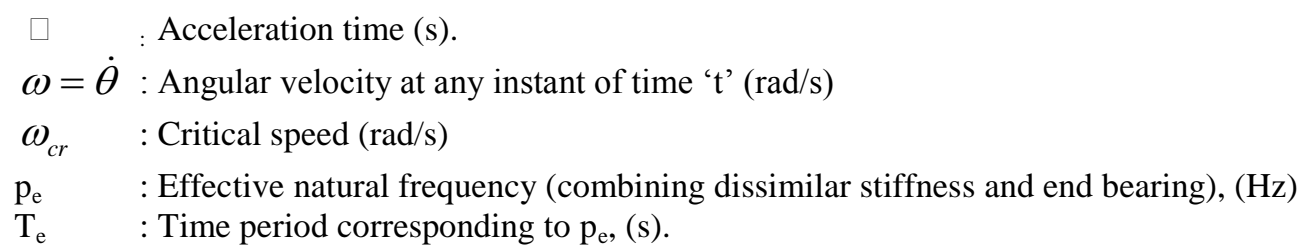

\section{INTRODUCTION}

Resonant vibrations in rotating machineries continue to pose serious problems to the engineer as vibration failures may cause disaster. Study of flexural vibrations of shaft-rotor systems is a subject of current interest where high speeds can provide enhanced power-to-weight ratio. The natural frequencies of a rotating machine actually differ from non-rotating ones because of the gyroscopic effect. High amplitude vibrations caused by rotation often occur at frequencies equal to the natural frequency of the rotor, called the critical speed and also at its submultiples and multiples [1,2,3,4,19].

An accurate prediction of dynamic behaviour is immensely valuable to the designer of modern high speed rotating machinery. To achieve this, a comprehensive mathematical model has to be constructed. Many rotors are symmetric and nearly rigid making the task of modeling and analysis somewhat simpler. Some rotors, on the other hand, are asymmetric and flexible; with the result, much complication is introduced in building the mathematical model and its analysis. Further, high speed flexible systems with large disks set up gyroscopic and rotatory inertia couples that introduce new and complex phenomena.

In general, the question of critical speeds of rotor systems has been addressed by many authors [5, 19-21]. The frequency equations and critical speeds of a straight circular rotor were obtained by Eshleman and Eubanks [5] who included the transverse shear, rotatory inertia and gyroscopic moments together with continuous shaft effects (distributed mass and elasticity) in their model. Research on rotor dynamics over the past three decades has been on improved models with the expedient of finite element formulation in some cases [6,8-12]. Ozguven and Ozkan [8], while considering the combined effect of shear deformation and internal damping, showed that requisite damping can suppress instability in homogeneous rotor-bearing systems.

The question of assessing dynamics stability becomes essential for ensuring better designs of rotor-systems and operational safety. The effects of bearing and shaft asymmetries on the stability of rotor has been reported by Ganesan [9]. Wettergren and Olsson [10] considered a horizontal rotor with a flexible shaft supported in flexible bearings and found that major instabilities appear near the imbalance resonance and remarked that the resonances due to gravity near one half of the major critical could be reduced with enhanced material damping. Rajalingham et al [15] considered the influence of external damping on the stability and dynamic response of single disk horizontal rotors with anisotropic bending stiffness characteristics

Campos et.al [19] reported a study on the dynamics of a Jeffcott rotor through Bond Graph formulation which provides for modelling of various nonlinear and multi-energetic systems. They validated their results through experiments on a carefully designed test setup obtaining a good agreement between the model predictions and the measured response

Chang and Cheng [22] considered the dynamics of a rotating shaft-disk system and particularly addressed the question of instability. They determined the stability criteria based on linear equations of motion and obtained analytical expressions for the radius of synchronous whirling. Chattoraj et. al [11] considered a highly flexible vertical Jeffcott rotor and noted interesting evolution of whirl when coriolis forces are included. Here, the phase-portraits did not settle on a single trajectory.

Very recently, Tammi [20] has reported active vibration control methods with a cascaded configuration controller employing inner feedback and outer feed forward loops to filter a sinusoidal reference signal in such a way that it acts against the disturbing rotor motions. The arrangement worked successfully with electromagnetic actuators built by modifying a magnetic bearing. The work is very elaborate where several control algorithms were tried. A good vibration damping performance was achieved over the entire operating range.

Panovko [12] has discussed several important aspects of rotor dynamics in his text book on Elastic Vibrations. Biezeno and Grammel [13] have produced a masterpiece work on rotor dynamics and more recently, Chong-Won-Lee [14] has on paper a fine reference manual on the subject. Den Hartog [18] in his text book on Mechanical Vibrations gives an excellent account of rotor dynamics especially clarifying the physics of gyroscopic couple.

In the present work, the authors consider the practical case of an overhanging high inertia horizontal rotor on a flexible isotropic shaft with an asymmetric end bearing to provide distinctly different elastic and damping characteristics in two fixed reference directions. The analysis includes the effects of rotatory inertia, gyroscopic couple, coriolis force and internal and external damping giving rise to a four degree-of-freedom model whose equations of motion are a set of ordinary differential equations with variable coefficients.

\section{DEVELOPMENT OF A PHYSICAL MODEL}

The phenomenology relating to rotor dynamics is complex and often counter-intuitive. Despite much study by several investigators over the past century lacunae still exist. The intent of this work is to develop a model capable of explicating the 
evolution of the whirl phenomenon and instability. The most common event of whirl - the synchronous whirl - forms the subject matter of the current investigation. A single disk on a flexible, horizontal cantilever shaft with a resilient orthotropic constraint (bearing) at the free end constitutes the proposed physical model and this configuration can accommodate the following notable actions:

Centrifugal forces due to imbalance, (2) Coriolis force, (3) Gyroscopic couple, (4) Elastic and Dissipative forces, (5) Gravity force
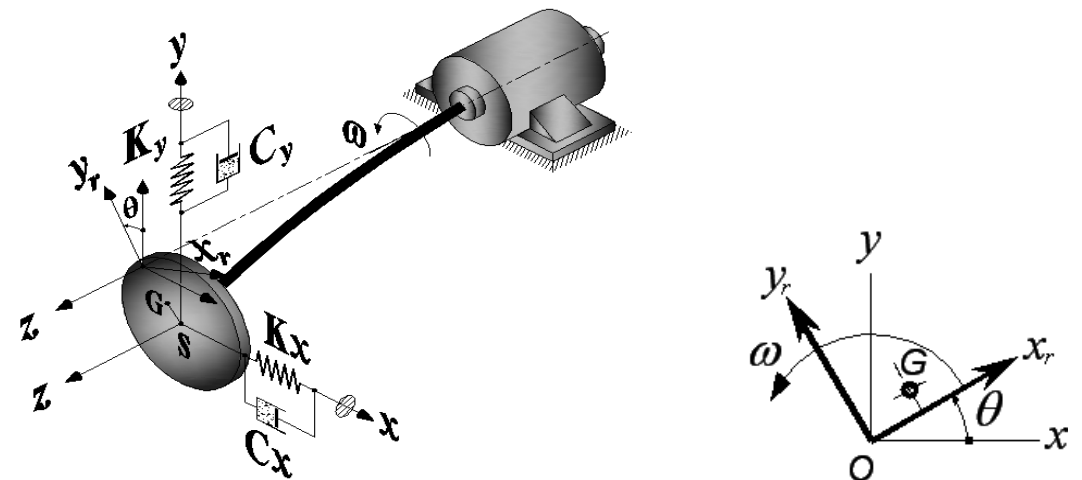

Fig.1: The Cantilever Rotor Model

Fig.2: Fixed and Rotating Reference Frames

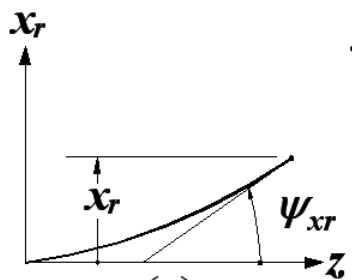

(a)

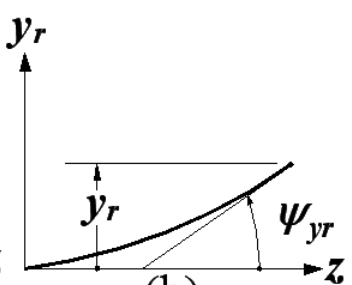

(b)

Fig.3(a, b): Projection of Slope-Deflection Pattern on rotating reference planes

Figure1 shows the overhung (cantilever) rotor model where the free end of the shaft is constrained by an orthotropic bearing providing additional elastic and dissipative supporting forces with fixed directions, while the first four of the above five types of actions rotate with the shaft-rotor system. The fifth force (i.e. gravity) also has a fixed direction. The energy to sustain the motion is provided by a motor with high inertia rotating parts so as to form a uniform speed source which is not readily affected by the kinetics of the rotor. In a large majority of cases, synchronous whirl has been reported by past observers [22]. In our model we then consider the rotor disk to execute a common rotational velocity with the shaft. For ease of keeping track of the various actions we propose a stationary $(\mathrm{x}, \mathrm{y})$ and a rotating $\left(\mathrm{x}_{\mathrm{r}}, \mathrm{y}_{\mathrm{r}}\right)$ Reference Frames as shown in fig.2. During the process of vibrations, the shaft laterally deflects in the $\mathrm{x}_{\mathrm{r}}-\mathrm{z}$ and $\mathrm{y}_{\mathrm{r}}-\mathrm{z}$ planes also producing the corresponding slopes $\psi_{\mathrm{xr}}$ and $\psi_{\mathrm{yr}}$ as shown in fig.3(a, b).

The co-ordinates of ' $G$ ' (the centre of mass of the disk) are expressible both in terms of the fixed and the rotating Reference Frames of fig.2. The following two transformation equations relate these two co-ordinate systems:

$$
\left\{\begin{array}{l}
x \\
y
\end{array}\right\}=\left[\begin{array}{cc}
\cos \theta & -\sin \theta \\
\sin \theta & \cos \theta
\end{array}\right]\left\{\begin{array}{l}
x_{r} \\
y_{r}
\end{array}\right\} \ldots \ldots . .(1) \text { and, }\left\{\begin{array}{l}
x_{r} \\
y_{r}
\end{array}\right\}=\left[\begin{array}{cc}
\cos \theta & \sin \theta \\
-\sin \theta & \cos \theta
\end{array}\right]\left\{\begin{array}{l}
x \\
y
\end{array}\right\}
$$

The equations of motion are formulated in the rotating Reference Frames (fig.2) for two reasons: first - to conveniently observe the effects of Coriolis, gyroscopic and elastic forces which rotate with the rotor system and second - to have expressions for the moment of inertia terms, especially for asymmetric rotors, as time-independent.

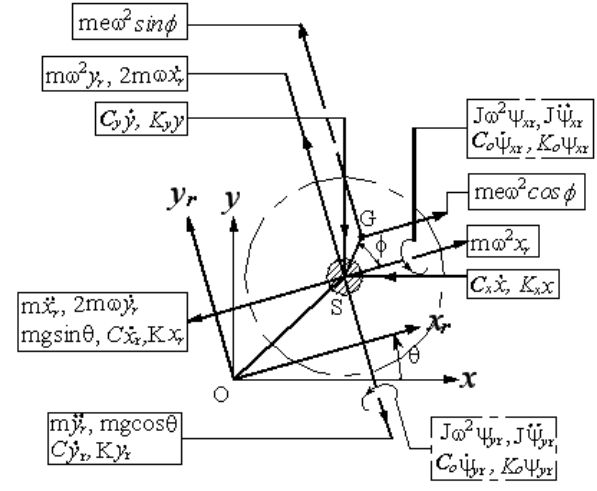

Fig.4: Forces and Moments on the Rotor System 
Figure 4 shows the end view of the rotor system where $\mathrm{O}$ corresponds with the bearing centre line and $\mathrm{S}$ is the deflected shaft centre $(\mathrm{OS}=$ shaft deflection) where $\mathrm{G}$ represents the centre of mass of the disk ( $\mathrm{SG}=$ eccentricity). The angle $\theta=\omega \mathrm{t}$ for a steady synchronous whirl where $\omega$ represents the constant rotary speed of the shaft. Fig.4 lists all the action components which will help us to formulate the four equations of motion (two each for lateral deflection and slope). It is notable that $J \ddot{\psi}_{x r}$ and $J \ddot{\psi}_{y r}$ are the rotatory inertia terms and following Den Hartog [18] $J \ddot{\psi}_{x r} \omega^{2}$ and $J \ddot{\psi}_{y r} \omega^{2}$ are recognized as gyroscopic actions, valid for small slopes. For large slope angles the gyroscopic terms shall be replaced by $J \omega^{2}\left(2 \sin \psi_{x r}-0.5 \sin 2 \psi_{x r}\right)$ and $J \omega^{2}\left(2 \sin \psi_{y r}-0.5 \sin 2 \psi_{y r}\right)$ in the $\mathrm{x}_{\mathrm{r}}-\mathrm{z}$ and $\mathrm{y}_{\mathrm{r}}-\mathrm{z}$ planes respectively.

Before proceeding with the mathematical formulation it will be instructive to note that the critical speed of a lumped mass cantilever shaft considering forward precession (gyroscopic effect), is given by:

$\omega_{c r}=\left[\frac{2 E I}{m L^{3}}\left\{\left(3-\frac{1}{D}\right)+\sqrt{\left(3-\frac{1}{D}\right)^{2}+\frac{3}{D}}\right\}\right]^{1 / 2}$

where $D=\frac{J}{m L^{2}}$

This speed is obviously higher than the natural frequency, $\mathrm{p}=\sqrt{\frac{3 E I}{m L^{3}}}$ of the non-rotating cantilever.

\section{FORMULATION}

We assume that the drive motor has the requisite capacity to maintain a constant spin in the rotor eliminating the need for tracking the torsional motion.

Applying d' Alembert's principle under dynamics equilibrium and referring to fig.4, the equations of motion for the system (small slopes) can be written as follows in the rotating reference frames:

$M \ddot{q}+\left(C_{i}+C_{e}+A\right) \dot{q}+\left(K_{i}+K_{e}+E+B+G\right) q=f$

Where:

$M=\left[\begin{array}{cccc}m & 0 & 0 & 0 \\ 0 & m & 0 & 0 \\ 0 & 0 & J & 0 \\ 0 & 0 & 0 & J\end{array}\right], \quad C_{i}=\left[\begin{array}{cccc}C & 0 & 0 & 0 \\ 0 & C & 0 & 0 \\ 0 & 0 & C_{0} & 0 \\ 0 & 0 & 0 & C_{0}\end{array}\right], \quad C_{e}=\left[\begin{array}{cccc}C_{11} & -C_{12} & 0 & 0 \\ -C_{12} & C_{11} & 0 & 0 \\ a_{1} C_{11} & -a_{1} C_{12} & 0 & 0 \\ -a_{1} C_{12} & a_{1} C_{11} & 0 & 0\end{array}\right]$,

$K_{i}=\left[\begin{array}{cccc}K & 0 & 0 & 0 \\ 0 & K & 0 & 0 \\ 0 & 0 & K_{0} & 0 \\ 0 & 0 & 0 & K_{0}\end{array}\right], \quad K_{e}=\left[\begin{array}{cccc}K_{11} & -K_{12} & 0 & 0 \\ -K_{12} & K_{11} & 0 & 0 \\ a_{1} K_{11} & -a_{1} K_{12} & 0 & 0 \\ -a_{1} K_{12} & a_{1} K_{12} & 0 & 0\end{array}\right], \quad E=\omega\left[\begin{array}{cccc}-C_{12} & -C_{11} & 0 & 0 \\ C_{11} & C_{12} & 0 & 0 \\ -a_{1} C_{12} & -a_{1} C_{11} & 0 & 0 \\ a_{1} C_{11} & a_{1} C_{12} & 0 & 0\end{array}\right]$,

$A=\left[\begin{array}{cccc}0 & 2 m \omega & 0 & 0 \\ -2 m \omega & 0 & 0 & 0 \\ 0 & 2 a_{1} m \omega & 0 & 0 \\ -2 a_{1} m \omega & 0 & 0 & 0\end{array}\right], \quad B=\left[\begin{array}{cccc}-m \omega^{2} & 0 & 0 & 0 \\ 0 & -m \omega^{2} & 0 & 0 \\ 0 & 0 & 0 & 0 \\ 0 & 0 & 0 & 0\end{array}\right], \quad G=\omega^{2}\left[\begin{array}{cccc}0 & 0 & a_{2} J & 0 \\ 0 & 0 & 0 & a_{2} J \\ 0 & 0 & J & 0 \\ 0 & 0 & 0 & J\end{array}\right]$.

where: $q=\left[\begin{array}{llll}x_{r} & y_{r} & \psi_{x r} & \psi_{y r}\end{array}\right]^{T}$, is the generalized coordinates vector of the rotor,

$\mathrm{f}=\mathrm{f}(\mathrm{t})+\mathrm{h}=m\left[\begin{array}{llll}-\sin \theta & -\cos \theta & -a_{1} \sin \theta & -a_{1} \cos \theta\end{array}\right]^{T}$

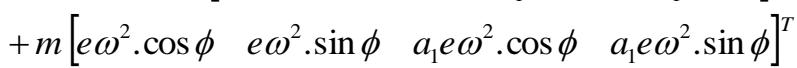

is an action vector composed of gravity, and centrifugal action due to eccentricity, where $\theta=\omega t$ for uniform synchronous whirl and $\phi$ defines the angular position of the mass centre of the disk relative to the rotating coordinate system.

Further,

$K_{11}=K_{m}+K_{n} \cos 2 \theta, \quad K_{12}=K_{n} \sin 2 \theta, \quad C_{11}=C_{m}+C_{n} \cos 2 \theta, \quad C_{12}=C_{n} \sin 2 \theta, \quad K_{m}=\frac{1}{2}\left(K_{x}+K_{y}\right), \quad K_{n}=\frac{1}{2}\left(K_{x}-K_{y}\right)$,

$C_{m}=\frac{1}{2}\left(C_{x}+C_{y}\right), C_{n}=\frac{1}{2}\left(C_{x}-C_{y}\right)$ and $a_{1}, a_{2}$ are coefficients such that :

force $\times a_{1}=$ moment equivalent of force ( producing slope) and moment $\times a_{2}=$ force equivalent of moment (producing deflection).

Equation (4) can be expanded to the following set after some mathematics:

$\ddot{x}_{r}+2\left[\zeta p+\zeta_{m} p_{m}\left(1+\mu_{c} \cos 2 \theta\right)\right] \dot{x}_{r}+2\left(\omega-\zeta_{m} p_{m} \mu_{c} \sin 2 \theta\right) \dot{y}_{r}+\left[p^{2}-\omega^{2}+p_{m}^{2}\left(1+\mu_{k} \cos 2 \theta\right)-2 \zeta_{m} p_{m} \mu_{c} \omega \sin 2 \theta\right] x_{r}-$

$\left[\mu_{k} p_{m}^{2} \sin 2 \theta+2 \zeta_{m} p_{m} \omega\left(1+\mu_{c} \cos 2 \theta\right)\right] y_{r}+a_{2} \rho^{2} \omega^{2} \psi_{x r}+g \sin \theta-e \omega^{2} \cos \phi=0$ 
Where: $p=\sqrt{\frac{K}{m}}, K=\frac{3 E I}{L^{3}}, p_{m}=\sqrt{\frac{K_{m}}{m}}, \mu_{k}=\frac{1-r_{k}}{1+r_{k}}, r_{k}=\frac{K_{y}}{K_{x}}, \mu_{c}=\frac{1-r_{c}}{1+r_{c}}, r_{c}=\frac{C_{y}}{C_{x}}, \rho=\sqrt{\frac{J}{m}}, \zeta=\frac{C}{2 \sqrt{K \cdot m}}, \zeta_{m}=\frac{C_{m}}{2 \sqrt{K_{m} . m}}$.

$\ddot{y}_{r}+2\left[\zeta p+\zeta_{m} p_{m}\left(1-\mu_{c} \cos 2 \theta\right)\right] \dot{y}_{r}-2\left(\omega+\zeta_{m} p_{m} \mu_{c} \sin 2 \theta\right) \dot{x}_{r}+$

$\left[p^{2}-\omega^{2}+p_{m}^{2}\left(1-\mu_{k} \cos 2 \theta\right)+2 \zeta_{m} p_{m} \mu_{c} \omega \sin 2 \theta\right] y_{r}-\left[\mu_{k} p_{m}^{2} \sin 2 \theta+2 \zeta_{m} p_{m} \omega\left(1-\mu_{c} \cos 2 \theta\right)\right] x_{r}+$

$a_{2} \rho^{2} \omega^{2} \psi_{y r}+g \cos \theta-e \omega^{2} \sin \phi=0$

$\ddot{\psi}_{x r}+\left(v^{2}+\omega^{2}\right) \psi_{x r}+2 \zeta_{0} q \dot{\psi}_{x r}+\frac{2 a_{1}}{\rho^{2}} \zeta_{m} p_{m}\left(1+\mu_{c} \cos 2 \theta\right) \dot{x}_{r}-$

$\frac{a_{1}}{\rho^{2}}\left[\omega^{2}-p_{m}^{2}\left(1+\mu_{k} \cos 2 \theta\right)+2 \zeta_{m} p_{m} \mu_{c} \omega \sin 2 \theta\right] x_{r}+\frac{2 a_{1}}{\rho^{2}}\left(\omega-\zeta_{m} p_{m} \mu_{c} \sin 2 \theta\right) \dot{y}_{r}-$

$\frac{a_{1} p_{m}}{\rho^{2}}\left[\mu_{k} p_{m} \sin 2 \theta+2 \zeta_{m} \omega\left(1+\mu_{c} \cos 2 \theta\right)\right] y_{r}+\frac{a_{1}}{\rho^{2}} g \sin \theta-a_{1} e \omega^{2} \cos \phi=0$

Where: $\zeta_{0}=\frac{C_{0}}{2 \sqrt{K_{0} . J}}, K_{0}=\frac{E I}{L}, v=\sqrt{\frac{K_{0}}{J}}$.

And,

$\ddot{\psi}_{y r}+\left(v^{2}+\omega^{2}\right) \psi_{y r}+2 \zeta_{0} q \dot{\psi}_{y r}+\frac{2 a_{1}}{\rho^{2}} \zeta_{m} p_{m}\left(1-\mu_{c} \cos 2 \theta\right) \dot{y}_{r}-\frac{2 a_{1}}{\rho^{2}}\left(\omega+\zeta_{m} p_{m} \mu_{c} \sin 2 \theta\right) \dot{x}_{r}-$

$\frac{a_{1}}{\rho^{2}}\left[\omega^{2}-p_{m}^{2}\left(1-\mu_{k} \cos 2 \theta\right)-2 \zeta_{m} p_{m} \mu_{c} \omega \sin 2 \theta\right] y_{r}-\frac{a_{1} p_{m}}{\rho^{2}}\left[\mu_{k} p_{m} \sin 2 \theta-2 \zeta_{m} \omega\left(1-\mu_{c} \cos 2 \theta\right)\right] x_{r}+\frac{a_{1}}{\rho^{2}} g \cos \theta-a_{1} e \omega^{2} \sin \phi=0$

We next define eight state variables: $\mathrm{x}_{\mathrm{r}}, \mathrm{y}_{\mathrm{r}}, v_{x r}=\dot{x}_{r}, v_{y r}=\dot{y}_{r}, \psi_{x r}, \psi_{y r}, \Omega_{x r}=\dot{\psi}_{x r}, \Omega_{y r}=\dot{\psi}_{y r}$.

Now, equations (5) to (8) can be conveniently written as eight first order differential equations, compressed into the following matrix form:

$\{\dot{s}\}=[F]\{s\}+\{H\} u$

Where,

$\{s\}=\left[\begin{array}{llllllll}s_{1} & s_{2} & s_{3} & s_{4} & s_{5} & s_{6} & s_{7} & s_{8}\end{array}\right]^{T}$

$=\left[\begin{array}{llllllll}v_{x r} & x_{r} & v_{y r} & y_{r} & \Omega_{x r} & \psi_{x r} & \Omega_{y r} & \psi_{y r}\end{array}\right]^{T}$

$[\mathrm{F}]=\left[\alpha_{\mathrm{ij}}\right]_{8 \mathrm{x} 8}, \mathrm{u}=\mathrm{g}$ and $\{H\}=\left[\begin{array}{llllllll}H_{1} & H_{2} & H_{3} & H_{4} & H_{5} & H_{6} & H_{7} & H_{8}\end{array}\right]^{T}$

Where $H_{1}=-\sin \theta+\frac{e}{g} \omega^{2} \cos \phi, H_{3}=-\cos \theta+\frac{e}{g} \omega^{2} \sin \phi, H_{5}=-\frac{a_{1}}{\rho^{2}} \sin \theta+\frac{a_{1} e}{g} \omega^{2} \cos \phi, \quad H_{7}=-\frac{a_{1}}{\rho^{2}} \cos \theta+\frac{a_{1} e}{g} \omega^{2} \sin \phi$

and $H_{2}=H_{4}=H_{6}=H_{8}=0$.

Elements $\alpha_{\mathrm{ij}}$ of the $8 \times 8$ coefficient matrix are embedded in equations (5) to (8).

These first order differential equations are formidable in structure but they can be numerically handled by appropriate numerical algorithms.

\section{EXPERIMENTAL SETUP: : THE ROTOR TEST FACILITY}

The present investigation is intended to provide an experimentally validated mathematical model of a flexible shaft-rotor system that shall be of service in explicating the evolution and quizzical nature of the whirl phenomenon while positively aiding the design of high speed machines; accordingly, an instrumented test facility has been designed and constructed. The rotor test facility is shown in fig.5 (photograph). This is a system with a single isotropic disk mounted at the end of a circular cantilever shaft where high deflection and slope can exist. Further, the free end of the cantilever is subjected to constraints provided by an orthotropic bearing of changeable asymmetry ratio. The disk is carefully balanced on the shaft and calculated amount of unbalance masses could be added on a pitch circle $(50 \mathrm{~mm}$. diameter). The cantilever end is supported on a pair of identical rigid ball bearings while the free end constraint is provided through a self-aligning ball bearing. The asymmetry parameters $\square_{\mathrm{c}}$ and $\square_{\mathrm{k}}$ could be altered by changing the suspension springs. The driving motor is D.C. permanent magnet type with the rotatory inertia of the armature enhanced (about 12 times) by the addition of a balanced flywheel at the drive end. This provides a fairly uniform speed drive system. The motor drives the shaft through a flexible coupling to compensate for any unavoidable alignment error. The speed variation is achieved by an electronic controller which can control the motor speed in the range of $30 \mathrm{rpm}$ to $6000 \mathrm{rpm}$ and the speed is measured by a digital photo tachometer (laser type). The rotor's lateral motion is tracked by an orthogonal pair of contactless velocity sensors aligned on a rigid support. The sensor output is conditioned by a signal conditioner (filter and integrator) and sent to the cathode ray oscilloscope for display which were transferred to computer through NI DAQ interface. An arrestor, serving as an initializer of the state variables and time, can be 
fitted at the flexible bearing end to prevent the vibratory motion of the rotor system during spin up. After achieving the required speed, the arrestor can be released so that normal vibrational dynamics is realized.

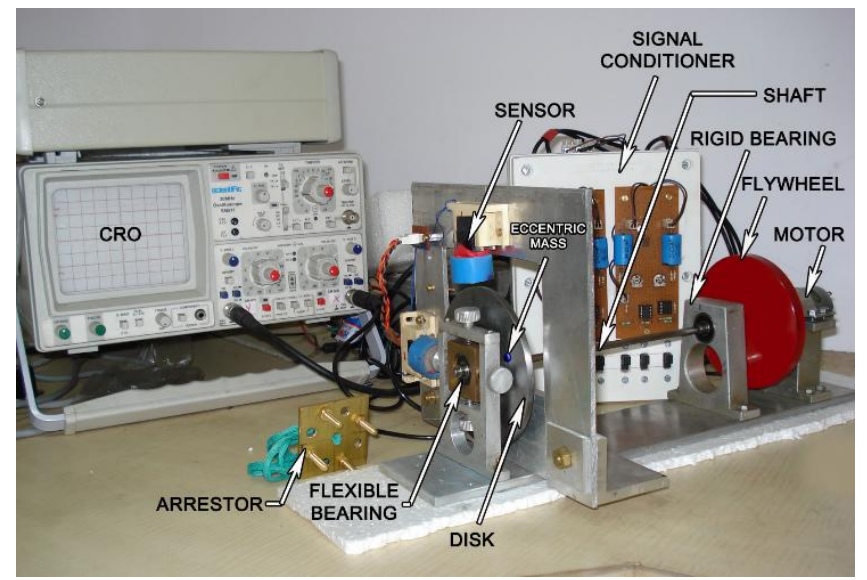

Table-1 provides the test data for the rotor system.

Table-1: Data for the Rotor system

\begin{tabular}{|l|l|l|}
\hline Features of the Set-up & Disk $_{1}$ & Disk $_{2}$ \\
\hline Diameter of disk, $\mathrm{d}_{1}(\mathrm{~mm})$ & 100 & 100 \\
\hline Thickness of disk, $\mathrm{t}(\mathrm{mm})$ & 5 & 2.5 \\
\hline Material of disk & $\mathrm{C} 20$ Steel & C20 Steel \\
\hline Mass of disk, $\mathrm{m}_{\mathrm{d}}(\mathrm{kg})$ & 0.308 & 0.157 \\
\hline Equivalent mass of Shaft-Disk system, $\mathrm{m}(\mathrm{kg})$ & 0.315 & 0.164 \\
\hline Lateral Bending Stiffness of Shaft, $\mathrm{K}(\mathrm{N} / \mathrm{m})$ & 2301 & 2301 \\
\hline Rotational Stiffness of Shaft $(\mathrm{wobble}), \mathrm{K}_{0}(\mathrm{~N}-\mathrm{m} / \mathrm{rad})$ & 30.68 & 30.68 \\
\hline M.I of Disk $($ diametral $), \mathrm{J}\left(\mathrm{kgm}^{2}\right)$ & $19.25 \times 10^{-5}$ & $9.8125 \times 10^{-5}$ \\
\hline Natural Frequency $($ bending $), \mathrm{p}(\mathrm{rad} / \mathrm{s})$ & 85.5 & 116 \\
\hline Natural Frequency $($ wobble $), \mathrm{q}(\mathrm{rad} / \mathrm{s})$ & 399.2 & 559.2 \\
\hline Horizontal Stiffness of Bearing, $\mathrm{K}_{\mathrm{x}}(\mathrm{N} / \mathrm{m})$ & 2200 & 2200 \\
\hline Horizontal stiffness of Bearing, $\left.\mathrm{K}_{\mathrm{y}} \mathrm{N} / \mathrm{m}\right)$ & 11000 & 11000 \\
\hline Centre of Gravity offset, e $(\mathrm{mm})$ & 0.50 & 0.50 \\
\hline
\end{tabular}

\begin{tabular}{|l|l|}
\hline \multicolumn{1}{|c|}{ Features of the Set-up } & Shaft \\
\hline Diameter of Shaft, $\mathrm{d}(\mathrm{mm})$ & 5 \\
\hline Length of Shaft, $\mathrm{L}(\mathrm{mm})$ & 200 \\
\hline Material of Shaft & C20 Steel \\
\hline Mass of shaft, $\mathrm{m}_{\mathrm{s}}(\mathrm{kg})$ & 0.0028 \\
\hline
\end{tabular}

\section{RESULTS AND DISCUSSIONS}

In the following illustrations we represent cases of sub-critical (at $\gamma=0.35$ and 0.36 ) to supercritical (at $\gamma=1.01$ ) rotors. The measured damping ratios is small (close to 0.01) which is typical for material damping, and all initial values of the state variables are kept zero and the eccentricity e $=0.5 \mathrm{~mm}$. Computer simulation is implemented using Runge-Kutta $4^{\text {th }}$ order algorithms and finally results are validated through experimentations.

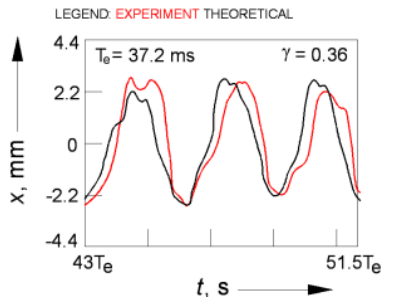

(a) Displacement-time plot

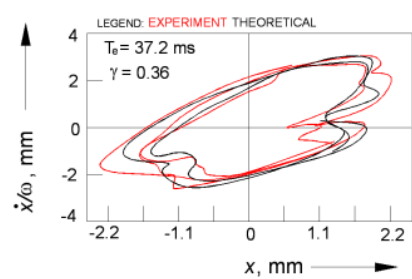

(b) Phase portrait 


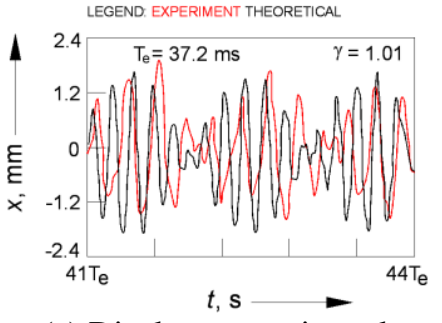

(c) Displacement-time plot

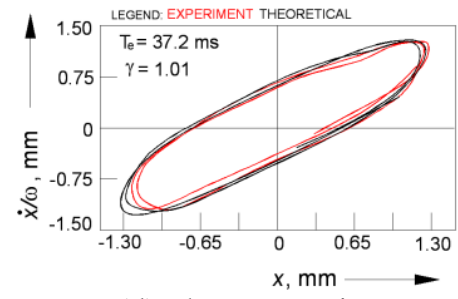

(d) Phase portrait

Fig.6 : Dynamic Response for $5 \mathrm{~mm}$ thick disk

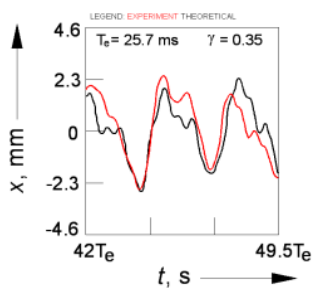

(a) Displacement-time plot

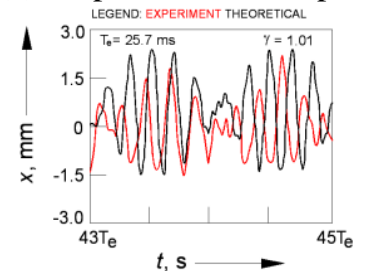

(c) Displacement-time plot

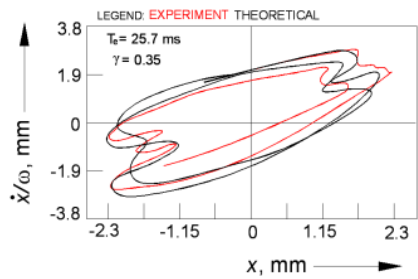

(b) Phase portrait

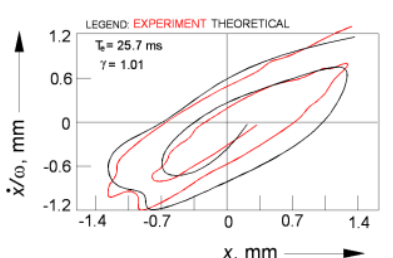

(d) Phase portrait

Fig.7: Dynamic Response for $2.5 \mathrm{~mm}$ thick disk

Figure 6 to 7 represent the displacement-time and phase plane plots (phase portraits) for two separate disk sizes (5mm and $2.5 \mathrm{~mm}$ ). Fig.6(b) shows the evolution of the phase plane plot for the motion of the shaft centre along the rotating $\mathrm{x}_{\mathrm{r}}$-axis with the frequency ratio $\gamma=0.36$ (sub-critical), damping ratio $\square \square=\square \square \square_{\mathrm{m}}=\square \square \square \square=0.01$ and $\mu_{\mathrm{c}}=\mu_{\mathrm{k}}=0.67$. The windings on the plot are due to a combination of harmonics at frequencies roughly conforming to p- $\square \square \square \square$ and p+The displacementtime graph, fig.6(a), clearly shows the presence of this multiple frequency oscillations which eventually settle to one of single frequency $\square \square$ de to damping of the transient at $\mathrm{t}>50 \mathrm{~T}_{\mathrm{e}}$. The phase portrait tends to a near elliptic band at higher time values which corroborates the persistence of the multiple frequency terms. Upon varying the initial conditions, it was observed that phase portraits settled to this band in the limit. The motion is stable.

In fig.6(c, d) a super critical $(\gamma=2)$ state in the verge of instability is described. The phase-plane trajectory settles to a nearly elliptic (band) shape. The time history shows a modulated wave shape which could be produced by two interfering harmonics of frequencies $2.5 \mathrm{p}_{\mathrm{e}}$ and $3 \mathrm{p}_{\mathrm{e}}$ whose conformity with $\square \square \mathrm{p}, \square \square$ and $\square \square\left(\right.$ ipe, $\mathrm{p}_{\mathrm{e}}, 2 \mathrm{p}_{\mathrm{e}}$ and $3 \mathrm{p}_{\mathrm{e}}$ ) is not found and the lowest frequency term is absent.

Figure 7(a, b, c, d) pertaining to $2.5 \mathrm{~mm}$ thick disk maintain the corresponding features of those for the $5 \mathrm{~mm}$ thick disk. While constructing fig.7 to 7 , the $\mathrm{x}_{\mathrm{r}}$-axis has been chosen for reference. If the $\mathrm{y}_{\mathrm{r}}$-axis were selected, like results, displaced in phase, would be obtained.

Comparison of the simulation results with the oscillograms (experimental results) shows a fair deal of correspondence which serve to validate the evolution of the motion pattern. Some departures are observable but they could not be refined as mechanical production (experimental set up) and instrumentation errors are inevitable, despite best care. We may say that the theory (i.e, model) is validated here.

Having studied some typical subcritical and supercritical responses described above it would be proper to address the question of stability of the system. The equations of motion of the system (eq.5 to eq.8) should answer this question, but the task of handling them analytically is far from simple because of their formidable structure. We have thus resorted to laborious scanning of the response over a range of $0.1 \mathrm{p} \leq \square \square \leq \square \square$ psing a very fine time step (approximately $10^{-4}$ times the shortest wave length of the vibrational motion). A non-dimensional deflection $\left(\frac{x_{r \max }}{\delta}\right)$ was tabulated against the non-dimensional frequency $\square \square$ and the results were plotted in fig.8 and fig.9. 

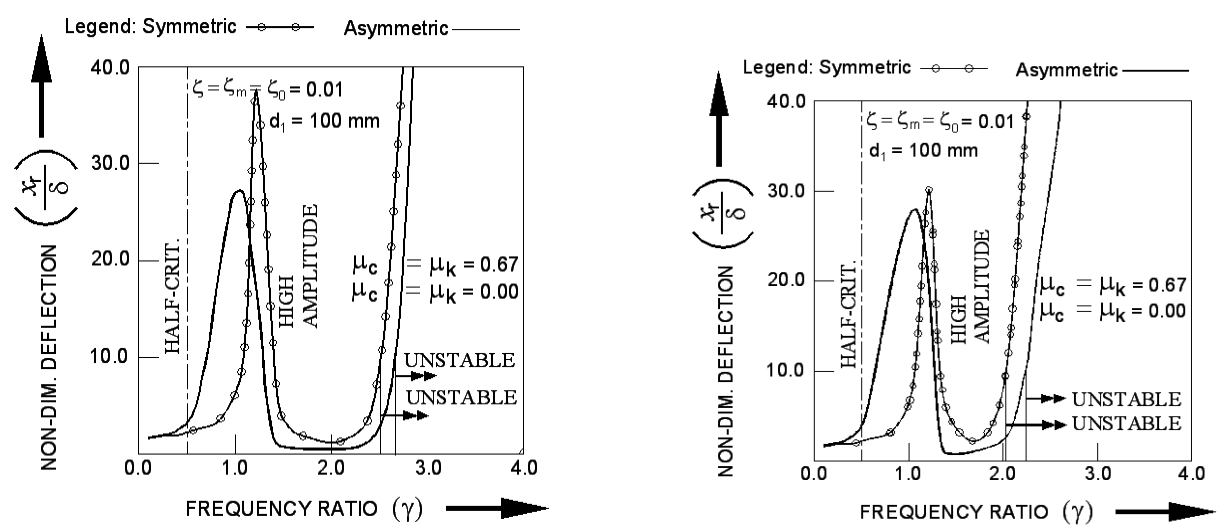

Fig.8 \& Fig.9: Stability Chart for a Rotor with Symmetric and Asymmetric end bearing and Gyro effect (5 mm \& $2.5 \mathrm{~mm}$ thick disk)

In stability charts for the two disks (fig.8 and 9), the asymmetry effect of the flexible end bearing are considered for two extreme cases: first, a high value of the asymmetry parameter $\mu_{\mathrm{c}}=\mu_{\mathrm{k}}=2 / 3(\approx 0.67)$ is shown in solid lines where $\mu_{\mathrm{c}}=\mu_{\mathrm{k}}=$ 0.01 and $\mathrm{d}_{1}=100 \mathrm{~mm}$ and second, a case of zero asymmetry is represented keeping all other parameters unchanged. In the former case in fig.8, a region spanning over $0.8<\gamma<1.2$ is a large amplitude zone containing the resonance location. A narrow zone of high amplitude, however, is observed in the latter case. With further increase of $\gamma$ the transient response becomes unstable at $\gamma>2.3$ where damping gives no benefit. For operational safety, we propose a limiting non-dimensional deflection $\left(\frac{x_{r \max }}{\delta}\right)=10$. Compared to symmetrical end bearings, a wider frequency band of high amplitude is observed in the high asymmetry case.

Figure 9 has features similar to fig. 8 . In this case, only the disk thickness is changed to $2.5 \mathrm{~mm}$ with a consequent rise in the natural frequency. No significant change in the shape of the stability boundaries is observable.

The rotor, operating beyond critical speed, has to cross this dangerous high amplitude zone with a large enough acceleration (or deceleration) so that the vibration amplitudes are not allowed to buildup violently and are made to stay put within a tolerable limit.

To determine the acceptable value of acceleration/deceleration, a study was made to track the variation of the normalized maximum displacement $\left(N_{\delta}=20 \cdot \log \left(\frac{x_{r, \max }}{\delta}\right)\right.$, in $\left.\mathrm{dB}\right)$ with the non-dimensional acceleration time $\left(\frac{\tau}{T_{p}}\right)$.

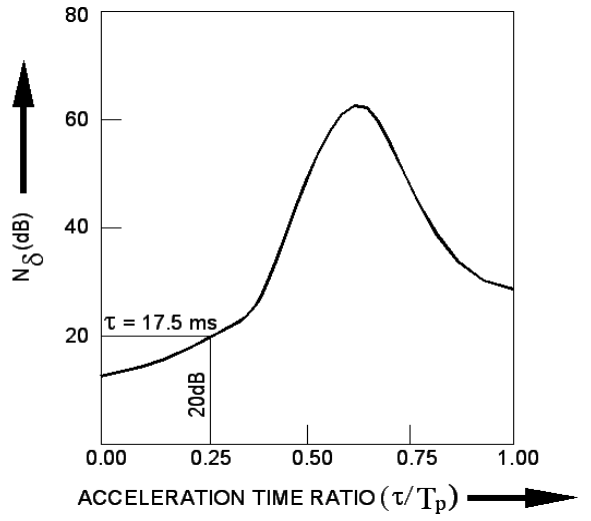

Fig.10: Variation of maximum displacement (for $5 \mathrm{~mm}$ disk), near critical zone

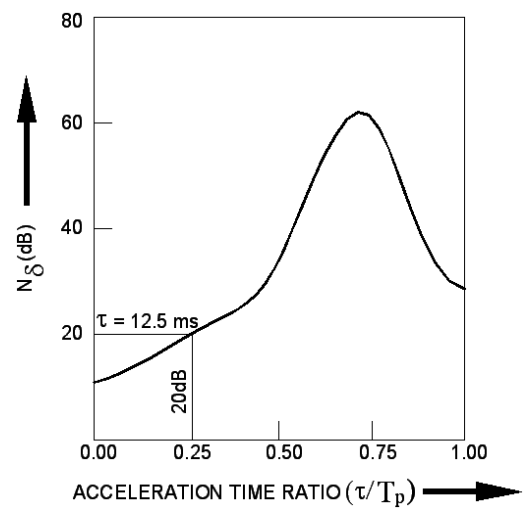

Fig.11: Variation of maximum displacement (for $2.5 \mathrm{~mm}$ disk), near critical zone

Figure 10 and fig. 11 depict the effect of uniform acceleration time $\tau$ to cross the critical resonance zone (from $0.8 \mathrm{p}_{\mathrm{e}}$ to $1.2 \mathrm{p}_{\mathrm{e}}$ ) on the vibration amplitude for the asymmetric disk. Fig. 10 shows a rapidly rising $\mathrm{N}_{\delta}$ value that moves from $20 \mathrm{~dB}$ at $\tau=$ $0.25 \mathrm{~T}_{\mathrm{p}}$ to $62.6 \mathrm{~dB}$ at $\tau=0.75 \mathrm{~T}_{\mathrm{p}}$ where $\mathrm{T}_{\mathrm{p}}$ is the time period of its oscillation. Fig. 11 shows the variation of $\mathrm{x}_{\mathrm{r}}$, max with time, $\tau$ for the $2.5 \mathrm{~mm}$ disk where the peak value of $\mathrm{x}_{\mathrm{r}}$, max reads $62 \mathrm{~dB}$. In both the disks, the critical zone should be crossed within $1 / 4$ natural oscillation $\left(\tau=0.25 \mathrm{~T}_{\mathrm{p}}\right)$. Considering the large $\left(2 \mathrm{krad} / \mathrm{s}^{2}\right.$ and $1 \mathrm{krad} / \mathrm{s}^{2}$ for $5 \mathrm{~mm}$ and $2.5 \mathrm{~mm}$ disks respectively) value of the required acceleration, a deflection arrestor must be provided to ease the process transition through the critical zone. 


\section{CONCLUSIONS}

The present study considers a rather flexible cantilever rotor as flexibility can enhance power-to-weight ratio. A fairly compliant orthotropic bearing at the free end is employed to reveal the effects of anisotropic support parameters. The resilient end support has been found to raise the range of stable operation speeds.

The phase-plane trajectories (Figs.6(b, d) and 7(b,d)) show a common feature: at the sub-critical and supercritical levels they appear as limit cycles. This is, however, not true as the same trajectory is never retraced and a band is formed instead to which the final responses are drawn when started with different initial conditions.

Operations near the critical speed $(\gamma=1)$, is observed to be dangerous during experimentation. In order to take the flexible rotor to its supercritical operating speed, the precarious critical zone should be crossed within $1 / 2$ natural oscillation or $0.5 \mathrm{~T}_{\mathrm{e}}$ that requires an average acceleration of $2300 \mathrm{rad} / \mathrm{s}^{2}$ and in the current test setup a power boost (for $20 \mathrm{~ms}$ ) of about $110 \mathrm{~W}$ becomes necessary. Here the maximum vibration amplitude can stay within a small value of $1.04 \mathrm{~mm}$ (fig.10). The same requirement is also valid for deceleration.

Doubtlessly, the required acceleration for transition through the critical zone is very high, and for a limited power drive motor $(50 \mathrm{~W})$ in the present setup a $250 \%$ short term power boost would be required. A displacement arrestor at the end bearing is a smarter alternative for speeding up to the supercritical operating point.

The inclusion of gyroscopic couple, rotatory inertia and Coriolis force raises the degrees of freedom of the rotor to four and the equations are formidable in structure. It is hoped that this could capture the features of the dynamics more comprehensively. A computer simulation became necessary and the theory has been validated by fairly agreeing experimental results. The study shows that rotors on asymmetric (orthotropic) bearings have superior stability characteristics. In the chosen example they can be run very smoothly in the supercritical range, $1.2<\gamma<2.2$. Nevertheless, Symmetric Bearings produce a lower bandwidth resonance zone which is easier to cross in acceleration/deceleration and are, therefore, preferable.

\section{ACKNOWLEDGEMENT}

The work of the present paper was supported by Dr. B. C. Roy Engineering College, Durgapur.

\section{REFERENCES}

[1] Karpenko, E., Pavlovskaia, E., and Wiercigroch, M., Bifurcation analysis of a preloaded Jeffcott rotor. Chaos, Solitons \& Fractals, J. of Sound and Vib.,15(2), 407-416 (2003).

[2] E.E. Pavlovskaia, E.V. Karpenko and M. Wiercigroch, Nonlinear dynamic interactions of Jeffcott rotor with preloaded snubber ring, J. of Sound and Vibration, 16 (1), 115-123 (2004).

[3] F.M. Dimentberg, Flexural Vibrations of Rotating Shafts, Butterworths, London (1961).

[4] R. Grybos, Gliwice, The effect of shear and rotary inertia of a rotor at its critical speeds, Applied Mechanics, 61,104-109 (1991).

[5] R. L. Eshleman and R. A. Eubanks, On the critical speeds of a continuous shaft-disk system, J. of Engineering for Industry, 89, 645-652 (1967).

[6] K. E. Rouch and J. S. Rao, A tapered beam finite element for rotor dynamics analysis, J. of Sound and Vib., 66, 119-140 (1979).

[7] T. C. Gmur and J. D. Rodrigues, Shaft finite elements for rotor dynamics analysis, J. of Vib. and Acoustics, 113, pp 482-493 (1991).

[8] H. Nevzat Ozguven, Z. Levent Ozkan, Whirl speeds and unbalance response of multi-bearing rotors using finite elements, J. of Vib., Acoustics, Stress, Reliability in Design, 106, 72-79 (1984).

[9] R. Ganesan, Effects of bearing and shaft asymmetries on the instability of rotors operating at near-critical speeds, Mechanism and Machine Theory, 35, 737-752 (2000).

[10] H.L. Wettergren and K.O. Olsson, Dynamic Instability of a Rotating Asymmetric Shaft with Internal Viscous Damping Supported in Anisotropic Bearings, J. of Sound and Vib., 195 (1), $75-84$ (1996).

[11] C. Chattoraj, S.N. Sengupta and M.C. Majumder, Dynamics of a Vertical Jeffcott Rotor, The Bulletin of Engineering and Science, 3 (2), 1-10 (2008).

[12] Ya Panovko, Elements of the Applied Theory of Elastic Vibrations, Mir Pub.,Moscow, 161-185 (1971).

[13] C.B. Biezeno, and R Grammel, Engineering Dynamics, Blackie \& Son, London, 180-259 (1954).

[14] Chong-Won Lee, Vibration Analysis of Rotors, Kluwer Academic Pub., Dordrecht, 1-54 (1993).

[15] C. Rajalingham, R.B. Bhat and G.D. Xistris, Influence of External Damping on the Stability and Resonance of a Rotor with Anisotropic Bearing Stiffness, Tribology Transactions, 393-398 (1993).

[16] P.V. Krishnamurthy, and S.K. Sen, Numerical Algorithms, Affiliated East-West Press, New Delhi, $441-454$ (1986).

[17] J. L. M. Morison and B. Crossland, An Introduction to Mechanics of Machines, ELBS, London, 79-81 (1966).

[18] J.P. Den Hartog, Mechanical Vibrations, McGraw-Hill Book Company, Inc, N.Y, 253-265 (1956).

[19] J. Campos et al, Rotordynamic Modelling Using Bond Graphs: Modelling the Jeffcott Rotor, IEEE Transactions on Magnetics, 41 (1), 274-280 (January 2005).

[20] K. Tammi, Active Control of Rotor Vibrations by Two Feedforward Control Algorithms, J. of Dynamics Systems, Measurement and Control (ASME), 131, 051012-1 to 051012-10 (September 2009).

[21] L. Banakh and A. Nikiforov, Backward Whirl Of Unbalanced Shaft At Elastically Constrained Journal Bearings And Floating Seals Due To Mechanical Contact, Proceedings of the $7^{\text {th }}$ International Scientific and Practical Conference, 234-240, (2009).

[22] C. O. Chang and J.W. Cheng, Non-Linear Dynamics and Instability of Rotating Shaft-Disk System”, J. of Sound and Vib., 160 (3), 433-454, (1993). 